\title{
Socio-demographic Correlates of Pre-pregnancy Body Mass Index Among Low-income Women in Hawaii WIC Participating in a Randomised Trial
}

\section{Michelle Asinobi}

University of Hawai'i at Mānoa: University of Hawai'i at Manoa

\section{Cristina Palacios}

Florida International University

\section{Yanyan Wu}

University of Hawai'i at Mānoa: University of Hawai'i at Manoa

Jinan Banna ( $\nabla$ jcbanna@hawaii.edu )

University of Hawai'i at Manoa https://orcid.org/0000-0003-0695-1205

\section{Research article}

Keywords: pregnancy, Asian, nutrition intervention, socio-demographic, body mass index, text message

Posted Date: June 2nd, 2021

DOl: https://doi.org/10.21203/rs.3.rs-569025/v1

License: (c) (i) This work is licensed under a Creative Commons Attribution 4.0 International License.

Read Full License 


\section{Abstract}

Background: A healthy pre-pregnancy BMI fosters positive outcomes for both mother and infant both during and after pregnancy. To design interventions to promote a healthy pre-pregnancy BMI in lowincome women, it is important to understand correlates. The purpose of this study was to identify the socio-demographic correlates of pre-pregnancy body mass index (BMI) among low-income women.

Methods: Participants were low-income pregnant women $(n=83)$ in the Special Supplemental Nutrition Program for Women, Infants, and Children (WIC) program in Hawai'i enrolled in a four-month text message-based nutrition intervention program. Participants reported pre-pregnancy weight and height and completed a demographics questionnaire on age, race/ethnicity, education, employment status and number of children. Descriptive statistics (mean, standard deviation for continuous variables, and frequencies and proportions for categorical variables) were used to summarize the sample. Simply linear regression analyses were performed to examine if independent variables were associated with BMI before pregnancy.

Results: Among the 83 women, 33 (39.8\%) were 18-24 years old, 22 (26.5\%) were between 25 and 29, 19 (22.9\%) were $30-34$ and $9(10.8 \%)$ were $35-41$. A total of 18 (21.7\%) were Asian. Age and race/ethnicity were marginally associated with BMI before pregnancy. The age group 30-34 had the highest BMI before pregnancy $(p=0.06)$ and Asian had lower BMI before pregnancy than the other races/ethnicities $(p=0.01)$.

Conclusions: Being classified as Asian was associated with lower BMI before pregnancy, while those who were 30-34 years old had the highest pre-pregnancy BMI compared to other age groups. These sociodemographic factors should be taken into account when designing interventions to promote healthy weight in women of childbearing age.

Trial registration: The trial is registered on clinicaltrials.gov (NCT04330976). Date of registration April 2, 2020 (restrospectively registered). URL: https://clinicaltrials.gov/ct2/show/NCT04330976

\section{Background}

Pre-pregnancy body mass index (BMI) plays a key role in child and maternal health. Previous research has shown that being overweight or obese prior to pregnancy is associated with an increased risk of still birth, large for gestational age, macrosomia, admission to the neonatal intensive care unit and low birthweight (LBW), while being underweight is associated with an increased risk of preterm birth, small for gestational age, and LBW (1). Undesirable outcomes for the offspring after birth with suboptimal prepregnancy BMI have also been identified; for example, pre-pregnancy overweight/obesity increases the risk of subsequent offspring overweight/obesity (2). Pre-pregnancy BMI also affects maternal health. Prepregnancy overweight/obesity increases the risk for gestational diabetes mellitus, for example.

Overweight women often gain excessive weight during pregnancy; a previous study revealed that $60 \%$ of women entering pregnancy overweight gained above the recommended level (3). Designing programs to 
assist those who are at higher risk of excessive gestational weight gain (GWG) is important because pregnancy is a critical window of opportunity to prevent two generations of obesity.

Pre-pregnancy $\mathrm{BMI}$ is determined by various socio-economic factors. A previous study conducted in the Northern region of Belgium revealed that a high pre-pregnancy BMI was significantly associated with low maternal education, high maternal age and multiparity, belonging to ethnic minority groups and a lower professional state (4). Researchers have found that low-income women (those living at $\leq 150 \%$ of the poverty guideline) are more likely to enter pregnancy overweight, gain more than the recommended amount of weight during pregnancy, and experience a higher risk of poor postpartum weight gain (5). A qualitative study that surveyed women who are in the first half and the last half of pregnancy found that low-income women have additional barriers to accessing healthy foods and preventing excessive GWG. Low-income women face challenges such as knowledge, cravings, complex policies, and stigma of receiving assistance from the Special Supplemental Nutrition Program for Women, Infants, and Children (WIC) (6).

Racial and ethnic differences in GWG have been found to vary by pre-pregnancy weight class. A previous review of United States data suggests that in normal-weight classes, Hispanic and black women are more likely to gain excessive GWG than white women. Among obese/overweight women, no significant difference was found between minorities and white women. Although black and Hispanic women are more likely to start pregnancy overweight/obese, low GWG has been found to be more common in these groups. The same review stated that over $40 \%$ of minority women gain above the 2009 Institute of Medicine (IOM) upper GWG limit (7).

A healthy pre-pregnancy BMI fosters positive outcomes for both mother and infant both during and after pregnancy. Research is lacking on the factors to consider in addressing the issue of suboptimal prepregnancy BMI in some parts of the U.S., such as Hawaii. To design interventions to promote a healthy pre-pregnancy BMI in low-income women in this diverse state, it is important to understand correlates. The objective of this study was to identify the socio-demographic correlates of pre-pregnancy BMI among low-income women in the WIC program in Hawai'i.

\section{Methods}

All procedures were in accordance with the ethical standards of the responsible committee on human experimentation (institutional and national) and with the Helsinki Declaration of 1975, as revised in 2000. Informed consent was obtained from all participants included in the study. The current study is a secondary analysis of the existing dataset.

Participants in the current study were 83 women from O\ahu WIC clinics who were 10-20 weeks pregnant and enrolled in a four-month text message-based nutrition intervention. The participants reported pre-pregnancy weight and height and completed a demographics questionnaire on age, race/ethnicity, education, employment status and number of children. BMI was calculated as weight 
$(\mathrm{kg}) /$ height $\left(\mathrm{m}^{2}\right)$. Detailed methods have been reported elsewhere (8). This study adheres to CONSORT guidelines and includes a completed CONSORT checklist.

Descriptive statistics (mean, standard deviation for continuous variables, and frequencies and proportions for categorical variables) were used to summarize the sample. Simply linear regression analyses were performed to examine if independent variables were associated with BMI before pregnancy. Significance was established at the $5 \%$ level. Given the small sample size, we also consider that a $p$-value less than $10 \%$ a signal of statistical significance.

\section{Results}

Among the 83 women, 33 (39.8\%) were 18-24 years old, 22 (26.5\%) were between 25 and 29, 19 (22.9\%) were $30-34$ and 9 (10.8\%) were 35-41. A total of 18 (21.7\%) were Asian. The mean BMI before pregnancy was $30.1 \mathrm{~kg} / \mathrm{m} 2$ (SD: 5.72 ), among which $45.8 \%$ were obese. Age and race/ethnicity were marginally associated with BMI before pregnancy based on simple linear regression analysis. As illustrated by Fig. 1, the age group 30-34 had the highest BMI before pregnancy, which were $3.03 \mathrm{~kg} / \mathrm{m} 2$ higher than those who were $18-24$ ( $95 \% \mathrm{Cl}:-0.16$ to $6.23, \mathrm{p}=0.062)$. BMI before pregnancy for Asians were $3.63 \mathrm{~kg} / \mathrm{m} 2$ lower than the other races/ethnicities ( $95 \% \mathrm{Cl}: 0.73$ to $6.52, \mathrm{p}=0.014$ ).

\section{Discussion}

The results of this study revealed a relationship between both race/ethnicity and age and pre-pregnancy BMI. This was the first study to examine socio-demographic correlates of pre-pregnancy BMI among lowincome women exclusively in Hawaii, which is of importance given the health disparities low-income groups continue to face in the state. Previous studies have also elucidated the relationship between race/ethnicity and other demographic characteristics and prepregnancy BMI. A study conducted in New York City indicated that black, Hispanic women and women who live in neighborhoods of low socioeconomic status are more likely to have a higher pre-pregnancy BMI than non-Hispanic white women (9). The authors also reported that black and Hispanic women with a college education and women living in low-income neighborhoods are more likely to have excessive GWG. White women are less likely to have excessive GWG according to the findings. Of note, the methods differed from those of the current study. The researchers collected data through birth certificates which contain information on maternal socio-demographic (race/ethnicity, education, age, zip code of residence, Medicaid status) and maternal and infant birth data (weight-gain, medical conditions, gestational age, parity), while data in the current study were collected through self-report. Socioeconomic status was indicated with education and neighborhood in Huynh et al.'s study, while the current study used income. Unlike the current study, Huynh et al.'s study did not include Asians or Native Hawaiians.

Another study which examines the race/ethnic disparities in the prevalence of pre-pregnancy obesity was conducted using data from the US Standard Certificate of Live Birth available through the Centers for Disease Control and Prevention (10). This study included 50 states and the District of Columbia between 
the years of 2012-2014. Results indicated that Samoan women had the highest rate of pre-pregnancy obesity (60.2\%), followed by Native Americans/Alaskans (34.9\%), blacks (33.9\%), Hawaiians (32.4\%), Puerto Ricans (28.6\%), Mexicans (28.0\%) and whites (22.3\%). Chinese women had the lowest rate of prepregnancy obesity $(2.6 \%)$ along with other Asian subgroups. This aligns with findings in the current study, indicating that Asians had lower BMI before pregnancy than the other races/ethnicities. Of note, the study included more racial/ethnic categories than the current study. Although the study does not include income, residing in a non-metropolitan county may be an indicator of low socioeconomic status which is associated with a higher prevalence of pre-pregnancy obesity than those who reside in metropolitan counties.

Also of note, there was also a positive relationship between age and BMI before pregnancy, except in the highest age group of 35-40 years of age. A previous study of women in nine states, not including Hawaii, revealed different findings, with the highest prevalence of pre-pregnancy obesity in those who were 20 to 29 years of age (11). Another study of women in California revealed that more than $50 \%$ of the women who were overweight or obese before pregnancy were 25 to 34 years of age (12). In the current study, the results with regards to age may be explained by the smaller sample size in this age bracket, as there were only nine women.

Identifying socio-demographic characteristics associated with pre-pregnancy BMI may inform interventions to address the specific needs of diverse groups. The limitations of the study are that prepregnancy weight and height are self-reported. Also, findings may not be generalized to other low-income pregnant women. Participants in the study were enrolled in WIC and met the income criteria for participation in the WIC program. Participants were not randomly selected from WIC, and due to this, it is not possible to generalize findings to the broader population of women enrolled in WIC. It is also possible that women who agreed to participate in the intervention may differ in some way from women in WIC who did not, perhaps in terms of being particularly interested in staying healthy.

\section{Conclusions}

This study revealed a relationship between demographic characteristics and pre-pregnancy BMI. Age and race/ethnicity were marginally associated with BMI before pregnancy. These socio-demographic factors should be considered when designing interventions for promoting adequate GWG. If it is known that particular groups struggle to maintain a healthy weight prior to pregnancy, tailored interventions may be developed to promote healthy eating during this time through promotion of culturally-appropriate foods and habits.

\section{Abbreviations}

The Special Supplemental Nutrition Program for Women, Infants, and Children=WIC; body mass index=BMI; low birthweight=LBW; gestational weight gain=GWG; Institute of Medicine=IOM 


\section{Declarations}

\section{Ethics approval and consent to participate}

Our research was approved by the University of Hawaii institutional review board and all procedures followed were in accordance with the ethical standards of the IRB and the Helsinki Declaration of 1975, as revised in 2000. Informed consent was obtained from all participants being included in the study.

\section{Consent for publication}

Not applicable.

\section{Availability of data and materials}

The datasets during and/or analysed during the current study and supporting the findings are available from the corresponding author on reasonable request.

\section{Competing interests}

None of the authors report a conflict of interest related to research presented in this article.

\section{Funding}

The authors acknowledge funding received from the Mountain West Clinical Translational Research Infrastructure Network under a grant from the National Institute of General Medicine Sciences of the National Institutes of Health. The funders had no role in the design of the study; in the collection, analyses, or interpretation of data; in the writing of the manuscript, or in the decision to publish the results.

\section{Authors' contributions}

JB and CP designed the study. YW analyzed data. MA and JB wrote the manuscript. All authors edited the manuscript. JB supervised the research. All authors read, revised and approved the final manuscript.

\section{Acknowledgements}

They authors acknowledge the participants in the study, as well as the following individuals for their help with data collection: Dustin Valdez, Michael Wong, Tina Lau, Jaya Sevilla, Meilani Galace, Cherese Shelton, Jocelyn Owan, Jacqueline Waters, Nahian Chowdhury, Samantha Manson, and Emiri Hirayama. They also acknowledge Linda Chock (WIC Branch Chief), Iris Takahashi (WIC Clinic Operations Section Chief), Laura Morihara (Wahiawa WIC Coordinator), Wendy Baker (Wahiawa WIC Office Assistant), Jean Kanda (Leeward WIC Coordinator), Dawn Fujimoto-Redoble (Leeward WIC Nutritionist), and Christina Mariano (Pearl City WIC Coordinator) from the Hawai'i Department of Health WIC Services Branch. 


\section{References}

1. Liu P, Xu L, Wang Y, Zhang Y, Du Y, Sun Y, et al. Association between perinatal outcomes and maternal pre-pregnancy body mass index. Obes Rev. 2016;17(11):1091-102.

2. Yu Z, Han S, Zhu J, Sun X, Ji C, Guo X. Pre-pregnancy body mass index in relation to infant birth weight and offspring overweight/obesity: a systematic review and meta-analysis. PLoS One. 2013;8(4):e61627.

3. Chu SY, Callaghan WM, Bish CL, D'Angelo D. Gestational weight gain by body mass index among US women delivering live births, 2004-2005: fueling future obesity. Am J Obstet Gynecol. 2009;200(3):271.e1-7.

4. Bogaerts A, Van den Bergh B, Nuyts E, Martens E, Witters I, Devlieger R. Socio-demographic and obstetrical correlates of pre-pregnancy body mass index and gestational weight gain. Clin Obes. 2012;2(5-6):150-9.

5. Nunnery D, Ammerman A, Dharod J. Predictors and outcomes of excess gestational weight gain among low-income pregnant women. Health Care Women Int. 2018;39(1):19-33.

6. Anderson CK, Walch TJ, Lindberg SM, Smith AM, Lindheim SR, Whigham LD. Excess gestational weight gain in low-income overweight and obese women: a qualitative study. J Nutr Educ Behav. 2015;47(5):404-11 e1.

7. Headen IE, Davis EM, Mujahid MS, Abrams B. Racial-ethnic differences in pregnancy-related weight. Adv Nutr. 2012;3(1):83-94.

8. Holmes H, Palacios C, Wu Y, Banna J. Effect of a Short Message Service Intervention on Excessive Gestational Weight Gain in a Low-Income Population: A Randomized Controlled Trial. Nutrients. 2020;12(5).

9. Huynh M, Borrell LN, Chambers EC. Maternal education and excessive gestational weight gain in New York City, 1999-2001: the effect of race/ethnicity and neighborhood socioeconomic status. Matern Child Health J. 2014;18(1):138-45.

10. Singh GK, DiBari JN. Marked disparities in pre-pregnancy obesity and overweight prevalence among US Women by race/ethnicity, nativity/immigrant status, and sociodemographic characteristics, 20122014. J Obes. 2019;2019:2419263.

11. Kim SY, Dietz PM, England L, Morrow B, Callaghan WM. Trends in pre-pregnancy obesity in nine states, 1993-2003. Obesity (Silver Spring, Md). 2007;15(4):986-93.

12. Ratnasiri AWG, Lee HC, Lakshminrusimha S, Parry SS, Arief VN, DeLacy IH, et al. Trends in maternal prepregnancy body mass index (BMI) and its association with birth and maternal outcomes in California, 2007-2016: A retrospective cohort study. PLoS One. 2019;14(9):e0222458.

\section{Figures}


(a) BMI before pregnancy and Age

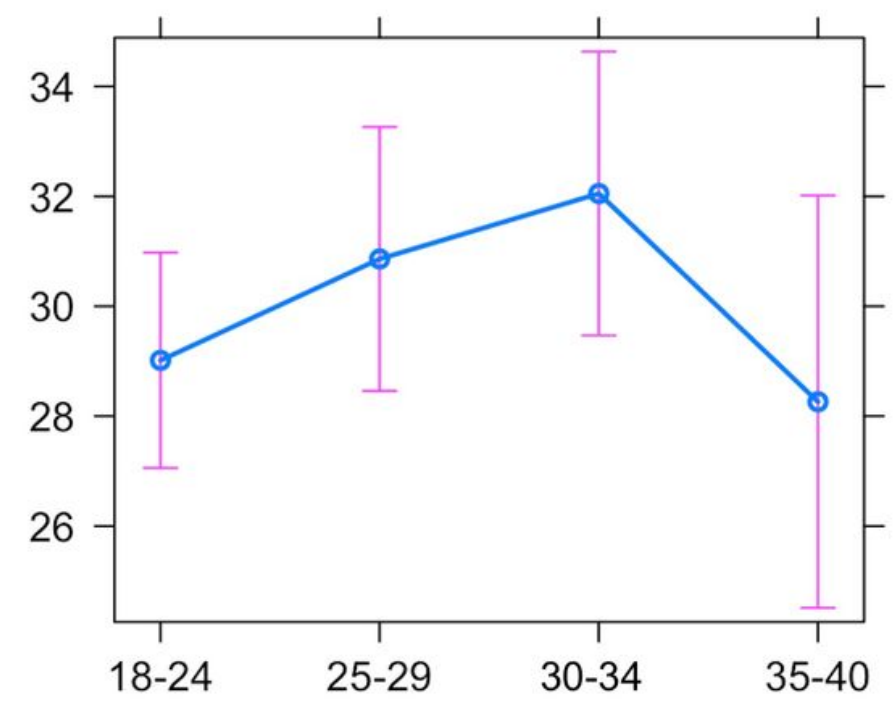

(b) BMI before pregnancy and Asian

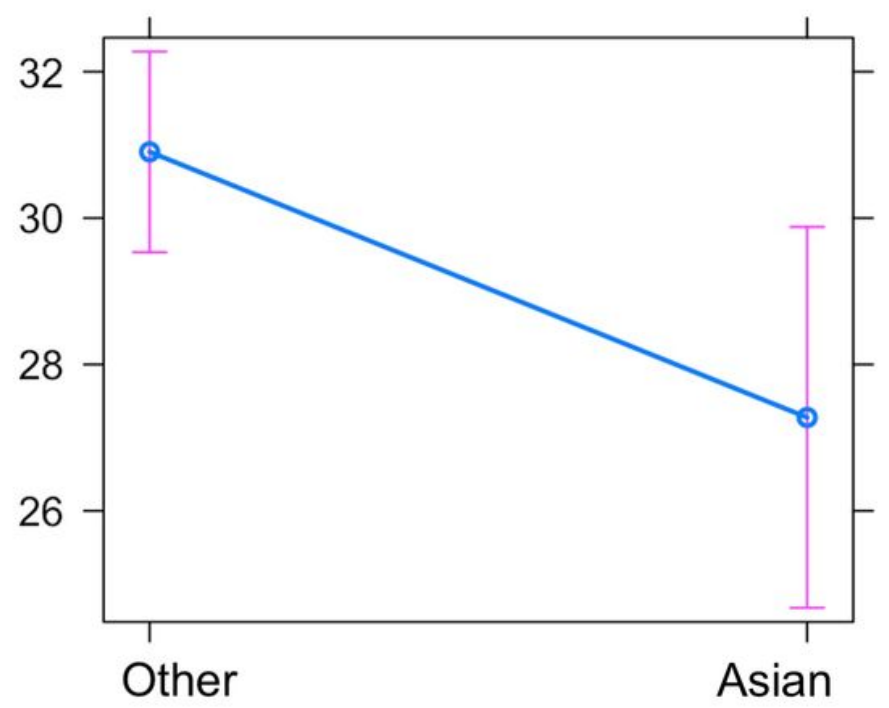

Figure 1

Association between BMI before pregnancy and age and race/ethnicity among low-income pregnant women $(n=83)$ in the Special Supplemental Nutrition Program for Women, Infants, and Children (WIC) program in Hawai'i

\section{Supplementary Files}

This is a list of supplementary files associated with this preprint. Click to download.

- CONSORT2010Checklist.doc 